\title{
A CONTRIBUIÇÃO DO COMPARTILHAMENTO DO CONHECIMENTO PARA O GERENCIAMENTO DE RISCOS EM PROJETOS: UM ESTUDO NA INDÚSTRIA DE SOFTWARE
}

\author{
KNOWLEDGE SHARING CONTRIBUTION TO PROJECT RISK \\ MANAGEMENT: A STUDY IN THE SOFTWARE INDUSTRY
}

\author{
Yóris Linhares Souza
}

UMA, MG, SERPRO, Brasil

Maria Celeste Reis Lobo Vasconcelos

Faculdade de Ciências Humanas de Pedro Leopoldo, Brasil

Valéria Maria Martins Judice

Universidade Federal de São João del-Rei (UFSJ), Brasil

George Leal Jamil

Fundação Mineira de Educação e Cultura FUMEC/MG, Brasil

\begin{abstract}
A study was conducted in the aim to identify whether and how knowledge sharing contributes, through instruments and behavioral aspects, to risk management. Initially, as the literature review was carried out, it was identified, on one hand, the main instruments and behavioral aspects that facilitate knowledge sharing and, on the other hand, the different approaches to risk management in software projects. Following that, the relationship between these two themes was established. Then, based on a case study in the software industry, an analysis was carried out and
\end{abstract}

Recebido em/Manuscript first received: 23/06/2008 Aprovado em/Manuscript accepted: 14/07/2009 Endereço para correspondência/ Address for correspondence

Yóris Linhares de Souza, mestre em Administração, especialista em Sistemas de Gestão de Empresas e graduado em Ciência da Computação. Professor de pós-graduação na UNA - MG, graduação na Faculdade Cotemig - MG e analista de sistemas no SERPRO. E-mail yoris.linhares@gmail.com

Maria Celeste Reis Lobo Vasconcelos, graduação em Engenharia Química, mestrado em Ciências Técnicas Nucleares, doutorado em Ciência da Informação e pos doutorado no Center for Research in Innovation Management - CENTRIM, na Inglaterra. Professora titular do Mestrado Profissional em Administração da Faculdade de Ciências Humanas de Pedro Leopoldo. E-mail: celestevasconcelos@gmail.com

Valéria Maria Martins Judice, PhD em Políticas de Ciência e Tecnologia (SPRU, University of Sussex, UK, 1997), mestre em Sociologia, especialista em Teoria Econômica e bacharel em Economia. É professora do Departamento de Ciências Administrativas de Contábeis, DECAC/ UFSJ, E-mail: valeriajudice@ufsj.edu.br

George Leal Jamil, graduação em Engenharia Elétrica, Mestrado em Ciência da Computação e doutorado em Ciência da Informação. Professor adjunto da Fundação Mineira de Educação e Cultura - FUMEC/BH. E-mail: gljamil@terra.com.br

ISSN online: 1807-1775

Publicado por/Published by: TECSI FEA USP - 2010 
applied to the development teams, aiming at understanding and perceiving what risk management activities are practiced in software projects, the behavioral factors that may favorably influence knowledge sharing on software projects risks and what instruments of knowledge sharing may contribute, at a certain degree, to risk management in projects.

Keywords: knowledge sharing, project management, risk management, software project.

\section{RESUMO}

Um estudo foi elaborado para identificar se e como compartilhamento do conhecimento contribui, por meio de instrumentos e aspectos comportamentais, para o gerenciamento de riscos em projetos. Inicialmente, buscou-se uma revisão da literatura, momento em que se identificaram, de um lado, os principais instrumentos e aspectos comportamentais facilitadores de compartilhamento do conhecimento e, de outro, as diferentes abordagens ao gerenciamento de risco em projetos de software. Em seguida, foi estabelecida a relação entre os dois temas. Depois se realizou uma análise com base em um estudo de caso na indústria de software aplicada às equipes de desenvolvimento, esperando-se entender e perceber quais as atividades de gerenciamento de riscos em projetos de software são praticadas, os aspectos comportamentais que podem influenciar favoravelmente para o compartilhamento de conhecimento sobre riscos em projetos de software e os instrumentos de compartilhamento do conhecimento que, em certo grau, podem contribuir para o gerenciamento de riscos em projetos.

Palavra-chave: compartilhamento do conhecimento, gerenciamento de projeto, gerenciamento de risco, projeto de software.

\section{INTRODUÇÃO}

Os crescentes e complexos desafios sobre o gerenciamento são resultados de condições ambientais que existiam antes, mas não com o grau existente hoje. Como parte da tentativa de encontrar uma solução interna, o gerenciamento de projetos é considerado uma das formas de melhor lidar com as atividades corporativas no intuito de obter melhor controle e uso dos recursos (Kerzner, 2003).

As organizações que gerenciam projetos lidam com riscos e necessitam gerenciá-los constantemente como forma de antecipar e minimizar o efeito de eventos que possam impactar negativamente nos objetivos dos projetos e, conseqüentemente, da organização.

Segundo Cooper (2003) uma das ferramentas mais poderosas no gerenciamento de riscos ao projeto é o conhecimento. E também segundo Tonet e Paz (2006), entre as estratégias que a literatura registra para gerar as condições de que as empresas necessitam para se manterem úteis e competitivas no mercado está a gestão e, em particular, o compartilhamento de conhecimento. Sob esses aspectos, espera-se que as organizações possam se apoiar no compartilhamento do conhecimento, mais especificamente em instrumentos e aspectos comportamentais citados por autores como Davenport e Prusak (1998), Nonaka e Takeuchi (1997), Prosbt et al. (2000), Bukowitz e Williams (2002), Gattoni (2004), Terra (2005) e Leonardi (2005) com o intuito de contribuir no processo de gerenciamento de riscos em projetos. 
Tendo em vista o exposto, embora os estudos e a literatura sobre o gerenciamento de projetos ainda explorem escassamente as conexões entre gestão de conhecimento e gestão de riscos em projetos, como suposição básica deste trabalho, considera-se que há uma relação efetiva entre o exercício de práticas de compartilhamento de conhecimento e o gerenciamento de riscos em projetos. A partir desta intuição sobre este relacionamento, faz-se necessário investigar empiricamente se e como se estabelecem conexões e suportes instrumentais entre duas áreas relacionadas, em uma área foco selecionada: a gestão de riscos em projetos de desenvolvimento de software.

Considerando estes direcionamentos, o artigo tem dois objetivos principais: (1) identificar no gerenciamento de projetos de software se, como contribui e que tipos de relações se estabelecem entre o compartilhamento de conhecimento e a gestão de riscos e (2) como é o processo de gerenciamento de riscos em projetos de desenvolvimento de software.

O que torna relevante o tema é a verificação de sua aplicação na indústria escolhida, a de software, devendo-se ao fato da importância dessa indústria. Segundo dados divulgados pela FUNSOFT (2007), a indústria de software de Belo Horizonte teve um dos setores econômicos que mais cresceu nos últimos cinco anos. Além disso, conta-se também com o alto nível de incerteza a que este ramo da indústria está exposto, se comparado a outros que trabalham com projetos.

Assim, espera-se que este artigo permita as organizações que lidam com projetos perceberem como é possível melhor gerenciá-los, visando diminuir a probabilidade e os impactos dos riscos aos quais estes são acometidos, aumentando as chances das organizações alcançarem seus objetivos.

Além desta seção introdutória, o artigo apresenta o referencial teórico dividido nas seções dois, três e quatro. Na seção dois, o compartilhamento do conhecimento é definido, os instrumentos e aspectos comportamentais facilitadores de compartilhamento do conhecimento são apresentados. Na seção três, é mostrada a importância do gerenciamento de projetos, e detalhados os processos de gerenciamento de riscos em projetos de qualquer natureza e de software e, por fim, as diversas abordagens da literatura sobre o tema são sintetizadas. Na seção quatro, tenta-se estabelecer o relacionamento entre o compartilhamento do conhecimento e sua contribuição para o gerenciamento de riscos em projetos. Após o referencial teórico, são apresentadas mais três seções. A quinta demonstra a metodologia de pesquisa, a sexta, a análise e interpretação dos resultados e a última, a conclusão do trabalho.

\section{COMPARTILHAMENTO DO CONHECIMENTO}

Argote e Ingram (2000) definem a transferência de conhecimento nas organizações como um processo pelo qual uma unidade (grupo, departamento ou divisão) é afetada pela experiência de outra. Embora a transferência de conhecimento em organizações envolva transferência no nível individual, o problema da transferência nas organizações transcende o nível individual para incluí-la em outros níveis, como grupo, departamento, etc. Nonaka e Takeuchi (1997) corroboram que o indivíduo é o elemento 
criador do conhecimento, a organização é o elemento ampliador e o grupo, o elemento de síntese do conhecimento.

Segundo Von Krogh et al. (2001), nas empresas, os indivíduos em geral compartilham conhecimentos sociais explícitos, arraigados e rotinizados, às vezes, formalizados por meio de procedimentos organizacionais para a execução de certa tarefa. Contudo, nem mesmo o conhecimento social explícito pode ser totalmente expresso por escrito ou convertido em rotinas, em que parte será compartilhada verbalmente ou mediante exemplos. Uma parcela substancial do conhecimento social também é tácita: envolve crenças compartilhadas sobre uma situação justificada, mas não explícita.

Para Polanyi (1966), citado por Magnani (2004), o termo “transferência” não é exatamente apropriado porque o conhecimento não pode ser manipulado como um objeto, o "recebedor" reconstrói sua versão do conhecimento recebido do "fornecedor" e muito do conhecimento de um especialista é tácito, e não pode ser articulado em contextos abstratos como numa entrevista. Assim, conforme Magnani (2004: 54), “a expressão 'transferência de conhecimento' vem sendo substituída pela expressão 'compartilhamento de conhecimento'”.

\subsection{Instrumentos de compartilhamento do conhecimento}

Um estudo de técnicas, métodos e instrumentos referenciados pelos diversos autores, como Terra (2005), Nonaka e Takeuchi (1997), Probst et al. (2000), Bukowitz e Williams (2002) e Davenport e Prusak (1998), permitiu elaborar uma síntese apontando seis instrumentos, com foco no compartilhamento do conhecimento, tendo como base as técnicas e os instrumentos de gestão de conhecimento descritos por Gattoni (2004):

1. Bancos de Competências: refere-se ao armazenamento das ligações entre os profissionais e às habilidades e competências que eles possuem.

2. Narrativas e histórias orais: significa contar histórias que podem ser extremamente úteis para uma organização, sobretudo pela vivência de profissionais mais experientes.

3. Cenários, Simulações e Protótipos: criar modelos de como a empresa deverá reagir e arquétipos de processos ou protótipos de produtos em função dos contextos apresentados.

4. Repositórios do conhecimento: normalmente tratam do conhecimento explícito estruturado na forma de documentos.

5. Comunidades de prática: surgem por consenso, quando várias pessoas vêem-se atraídas por uma força social e profissional que as impele a cooperarem.

6. Equipes multidisciplinares: agregar diferentes fontes de conhecimento ao redor de problemas específicos.

Segundo Gattoni (2004), existem diversos outros mecanismos para melhor administrar tanto a geração quanto a codificação e a transferência do conhecimento no interior das organizações. No entanto, segundo o que foi apurado pelo autor, a maior 
parte destes mecanismos alternativos se enquadra, de uma forma ou de outra, em pelo menos uma destas categorias, quando não de várias delas. Além disso, e de forma proposital, não foram abordadas as possíveis ferramentas de tecnologia da informação disponíveis para a automatização dos mecanismos de gestão do conhecimento.

\subsection{Aspectos facilitadores de compartilhamento do conhecimento}

Von Krogh et al. (2001) afirmam que o compartilhamento de conhecimento é um processo extremamente frágil, pois exige que as pessoas se exponham ao dividir e justificar seus valores e crenças pessoais perante outros. Este processo não se sujeita às técnicas de gestão tradicionais. Por este motivo, deve ser apoiado por várias atividades organizacionais que criem condições para que ele ocorra, apesar de barreiras e obstáculos. Essas barreiras ocorrem tanto no nível do indivíduo quanto no âmbito organizacional, de forma inter-relacionada.

Para Leonardi (2005), em um primeiro momento, o indivíduo precisa ter a iniciativa de compartilhar seu conhecimento e isso é fruto de um esforço pessoal e voluntário. Em segundo lugar, é preciso um campo no qual os indivíduos possam interagir uns com os outros. A autora analisou as bases da disposição em compartilhar conhecimento, que se fundamenta no comportamento do indivíduo, influenciado não só pelas suas características e valores pessoais, mas também moldado pelos valores do grupo e da organização onde atua. Segundo a autora, a literatura oferece um elenco de condições ou aspectos que podem influenciar favoravelmente o compartilhamento de conhecimento na organização e, sobretudo, a disposição para compartilhar conhecimento com base nas atitudes e percepções dos indivíduos. Esses aspectos podem advir de crenças e valores pessoais ou ser percebidos como valores pregados pela equipe onde este indivíduo atua ou mesmo pela organização. Entre os aspectos citados pela autora, cinco foram selecionados pela maior consonância com os outros autores como Von Krogh et al. (2001), Bukowitz e Williams (2002), Davenport e Prusak (1998), Probst et al. (2000) e Terra (2005):

1. Reconhecimento: percepção do indivíduo de que o compartilhamento de conhecimento é devidamente reconhecido.

2. Consciência da utilidade do conhecimento: consciência de que o conhecimento pode ter utilidade para outras pessoas na organização.

3. Reciprocidade: percepção de que, ao compartilhar um recurso, a outra parte estará disposta a retribuir com um conhecimento de mesmo valor.

4. Confiança: certeza de que o compartilhamento de conhecimento não trará danos a si mesmo, certeza quanto ao uso a ser feito do conhecimento compartilhado.

5. Relevância: percepção de que há ganhos pessoais relevantes ao se compartilhar conhecimento.

Tonet e Paz (2006) enfatizam que a realidade está mostrando às organizações a necessidade de estimular colaboradores e parceiros a compartilharem os conhecimentos que possuem. 
A percepção de que o conhecimento é fonte de riqueza e vantagem competitiva tem estimulado o interesse pela gestão do conhecimento em organizações que tratam de projetos (Pretorius; Steyn, 2005).

\section{GERENCIAMENTO DE RISCOS EM PROJETOS}

Para Alencar e Schmitz (2005), um projeto visa propiciar algum tipo de benefício ou vantagem competitiva. Apesar dos benefícios potenciais advindos do gerenciamento de projetos, infelizmente estes não podem ser alcançados sem transpor barreiras como a complexidade do projeto, requerimentos especiais dos clientes e mudanças de escopo, reestruturação organizacional, mudanças de tecnologia e riscos (PMI, 2004).

Segundo Kendrick (2003), em projetos, um risco pode ser qualquer evento indesejável associado com o trabalho e é o produto de dois fatores: os impactos esperados de um evento e a probabilidade de que o evento possa ocorrer. Em se tratando de riscos em projetos, o Project Management Institute, PMI (2004) define risco como um evento ou condição incerta sobre um objetivo do projeto, como tempo, custo, escopo ou qualidade.

As organizações que lidam com o desenvolvimento de software têm figurado em importância crescente economicamente. E para assegurar que o desenvolvimento e a manutenção do software sejam sistemáticos, disciplinados e qualificados, as organizações têm dado ênfase à aplicação do gerenciamento das atividades, do planejamento, da coordenação, da mensuração, do monitoramento, do controle e do nível de divulgação (Swebok, 2004).

Estes projetos de desenvolvimento de software estão expostos a incertezas das mais variadas origens e a gerência de riscos é a área de estudo que enfoca o planejamento e o acompanhamento dessas incertezas (Schwalbe, 2002). Alguns dos motivos são expostos por Leopoldino (2004): os projetos de software apresentam uma série de peculiaridades em relação a outros tipos de projetos; estão situados em ambiente de aplicação relativamente intensa de tecnologias, em campo de atuação impregnado de incerteza; por ser uma área relativamente recente, muitas das atividades feitas não têm precedentes em que se possam fundamentar certas decisões. E ainda, segundo Gusmão e Moura (2005), estes projetos estão sendo afetados por riscos que são inesperados, nãoplanejados ou ignorados simplesmente.

\subsection{Abordagens ao gerenciamento de riscos em projetos de software}

O PMI (2004), por meio do guia Project Management Body of Knowledge PMBoK, e a norma AS/NZS 4360:2004 ${ }^{1}$, como relatada por Ferreira (2006), tratam do 
gerenciamento de riscos para qualquer tipo de projeto. Ambos têm abordagens equivalentes se diferenciando no destaque dado a comunicação de riscos pela AS/NZS 4360:2004. Em resumo, o processo de gerenciamento de riscos contempla as seguintes atividades, conforme QUADRO 1.

QUADRO 1

Síntese das atividades do processo de gerenciamento de riscos em projetos

Gerenciamento de Riscos em projetos

\begin{tabular}{|c|c|c|}
\hline PMBoK & AS/NZS 4360:2004 & Descrição da atividade \\
\hline $\begin{array}{l}\text { Planejamento do } \\
\text { gerenciamento de riscos }\end{array}$ & $\begin{array}{l}\text { Estabelecimentodos } \\
\text { contextos }\end{array}$ & $\begin{array}{l}\text { decisão de como abordar, planejar e executar as } \\
\text { atividades de gerenciamento de riscos }\end{array}$ \\
\hline Identificação de riscos & Identificação de riscos & $\begin{array}{l}\text { identificar quais, onde, quando, por que e como os } \\
\text { eventos podem impedir, atrapalhar, atrasar ou } \\
\text { melhorar a consecução dos objetivos }\end{array}$ \\
\hline $\begin{array}{l}\text { Análise qualitativa e } \\
\text { quantitativa de riscos }\end{array}$ & $\begin{array}{l}\text { Análise e Avaliação de } \\
\text { riscos }\end{array}$ & $\begin{array}{l}\text { identificar e avaliar os controles existentes, } \\
\text { determinar as conseqüências e a probabilidade e, } \\
\text { por conseguinte, o nível de risco }\end{array}$ \\
\hline $\begin{array}{l}\text { Planejamento } \\
\text { respostas a riscos }\end{array}$ & Tratamento de riscos & $\begin{array}{l}\text { desenvolver estratégias e planos de ação } \\
\text { específicos e econômicos, para aumentar } \\
\text { oportunidades e reduzir as ameaças aos objetivos }\end{array}$ \\
\hline $\begin{array}{l}\text { Monitoramento } \\
\text { controle de riscos }\end{array}$ & $\begin{array}{l}\text { Monitoramento e análise } \\
\text { crítica }\end{array}$ & $\begin{array}{l}\text { acompanhamento dos riscos identificados, } \\
\text { monitoramento dos residuais, identificação dos } \\
\text { novos, execução de planos de respostas a eles e } \\
\text { avaliação da sua eficácia }\end{array}$ \\
\hline Comunicação implícita & Comunicação e consulta & $\begin{array}{l}\text { comunicar e consultar as partes em cada etapa do } \\
\text { processo de gerenciamento de riscos e em relação } \\
\text { ao processo como um todo }\end{array}$ \\
\hline
\end{tabular}

Fonte: Adaptado de PMI (2004) e Ferreira (2006).

Para Machado (2002), a aplicação dos conceitos e princípios de gerência de risco de outras disciplinas tem requerido adaptações para software. As adaptações realizadas fazem com que diferentes fontes tenham diferentes definições para escopo e atividades da gerência de risco. Segundo Gusmão e Moura (2005), diversas abordagens que apresentam um processo para a gerência de riscos são encontradas na literatura na área de desenvolvimento de software.

Gusmão e Moura (2005), Rocha e Belchior (2004) e Machado (2002) abordam o gerenciamento de riscos em projetos de software segundo alguns modelos, entre os quais se destacam o CMMI-SW (Capability Maturity Model Integration for Software); a norma ISO/IEC 12207 - Processos de Ciclo de Vida de Software e ISO/IEC TR 15504 Part 5: Assessment Model and Indicator Guidance; o RUP (Rational Unified Process) e o MSF (Microsoft Solutions Framework). As atividades do processo de gerenciamento 
de riscos em projetos de software, como relatado pelos autores, estão sintetizadas no Quadro 2.

\section{QUADRO 2}

Síntese das atividades do processo de gerenciamento de riscos em projetos de software

\begin{tabular}{|c|c|c|c|c|}
\hline \multicolumn{5}{|c|}{ Gerenciamento de Riscos em projetos de Software } \\
\hline CMMI-SW & RUP & \begin{tabular}{lc}
\multicolumn{2}{c}{ ISO/IEC } \\
12207 & ISO/IEC \\
$15504-5$ &
\end{tabular} & MSF & Descrição da atividade \\
\hline $\begin{array}{l}\text { Determinar a } \\
\text { origem e as } \\
\text { categorias de } \\
\text { riscos e } \\
\text { estabelecer } \\
\text { estratégias }\end{array}$ & $\begin{array}{l}\text { Desenvolver o } \\
\text { Plano de } \\
\text { Gerenciamento } \\
\text { de Riscos }\end{array}$ & $\begin{array}{l}\text { Estabelecer o } \\
\text { escopo da gerência } \\
\text { de risco }\end{array}$ & & $\begin{array}{l}\text { desenvolver o escopo da } \\
\text { gerência de risco que será } \\
\text { utilizada pelo projeto }\end{array}$ \\
\hline $\begin{array}{l}\text { Identificar } \\
\text { Riscos }\end{array}$ & $\begin{array}{l}\text { Identificar e } \\
\text { Avaliar os } \\
\text { Riscos }\end{array}$ & $\begin{array}{l}\text { Identificar } \\
\text { riscos }\end{array}$ & $\begin{array}{l}\text { Identificar } \\
\text { riscos }\end{array}$ & $\begin{array}{l}\text { identificar riscos para o projeto, } \\
\text { no início e durante a sua } \\
\text { execução }\end{array}$ \\
\hline $\begin{array}{l}\text { Priorizar, estimar } \\
\text { e classificar risco }\end{array}$ & $\begin{array}{l}\text { Identificar e } \\
\text { Avaliar os } \\
\text { Riscos }\end{array}$ & $\begin{array}{l}\text { Analisar e } \\
\text { priorizar } \\
\text { riscos }\end{array}$ & $\begin{array}{l}\text { Analisar } \\
\text { riscos }\end{array}$ & $\begin{array}{l}\text { avaliar a probabilidade, o } \\
\text { impacto, o tempo de } \\
\text { ocorrência, a causa e as } \\
\text { relações entre os riscos para } \\
\text { determinar sua prioridade }\end{array}$ \\
\hline $\begin{array}{l}\text { Desenvolver } \\
\text { Planos de redução } \\
\text { de risco }\end{array}$ & $\begin{array}{l}\text { Identificar e } \\
\text { Avaliar os } \\
\text { Riscos }\end{array}$ & $\begin{array}{l}\text { Definir a } \\
\text { Estratégia para a } \\
\text { gerência de } \\
\text { risco }\end{array}$ & $\begin{array}{l}\text { Planejar } \\
\text { riscos }\end{array}$ & $\begin{array}{l}\text { definir uma estratégia para } \\
\text { gerenciar um risco ou um } \\
\text { conjunto de riscos em nível de } \\
\text { projeto e organizacional e } \\
\text { construir planos que suportarão } \\
\text { a tomada de decisão e as ações }\end{array}$ \\
\hline $\begin{array}{l}\text { Implementar } \\
\text { Planosde redução } \\
\text { de risco }\end{array}$ & $\begin{array}{l}\text { Monitorar o } \\
\text { Status do } \\
\text { Projeto }\end{array}$ & $\begin{array}{l}\text { Definir } \\
\text { Métricaspara } \\
\text { riscos, implementar } \\
\text { a estratégia da } \\
\text { gerência de risco e } \\
\text { avaliar os } \\
\text { resultados da } \\
\text { estratégia da } \\
\text { gerência de risco }\end{array}$ & $\begin{array}{l}\text { Acompanhar } \\
\text { e Controlar } \\
\text { riscos }\end{array}$ & $\begin{array}{l}\text { monitorar a situação dos riscos } \\
\text { e as respostas, avaliar o } \\
\text { progresso esperado e o nível de } \\
\text { sucesso da estratégia, corrigir } \\
\text { ou evitar impacto. }\end{array}$ \\
\hline $\begin{array}{l}\text { Comunicação } \\
\text { implícita }\end{array}$ & $\begin{array}{l}\text { Comunicação } \\
\text { implícita }\end{array}$ & $\begin{array}{l}\text { Comunicação } \\
\text { implícita }\end{array}$ & $\begin{array}{l}\text { Comunicaçã } \\
\text { o implícita }\end{array}$ & \\
\hline
\end{tabular}

Fonte: Adaptado de Rocha e Belchior (2004), Machado (2002), Gusmão e Moura (2005).

O QUADRO 3 apresenta um mapeamento das diversas abordagens de 
gerenciamento de risco em projetos de software, tendo como base as atividades da área de conhecimento de gerenciamento de risco descritas pelo guia Project Management Body of Knowledge - PMBoK, conforme o PMI (2004) e a norma AS/NZS 4360:2004, citada por Ferreira (2006).

QUADRO 3 - Mapeamento das atividades do processo de gerenciamento de riscos em projetos de qualquer natureza e de software

\begin{tabular}{|c|c|c|c|c|c|}
\hline \multicolumn{2}{|c|}{$\begin{array}{l}\text { Gerenciamento de Riscos em } \\
\text { projetos }\end{array}$} & \multicolumn{4}{|c|}{ Gerenciamento de Riscos em projetos de Software } \\
\hline PMBoK & $\begin{array}{l}\text { AS/NZS } \\
4360: 2004\end{array}$ & CMMI-SW & RUP & \begin{tabular}{lc}
\multicolumn{2}{c}{ ISO/IEC } \\
12207 & ISO/IEC \\
$15504-5$ &
\end{tabular} & MSF \\
\hline $\begin{array}{l}\text { Planejamento } \\
\text { do } \\
\text { gerenciamento } \\
\text { de riscos }\end{array}$ & $\begin{array}{l}\text { Estabelecimen- } \\
\text { to dos } \\
\text { contextos }\end{array}$ & $\begin{array}{l}\text { Determinar a } \\
\text { origem e as } \\
\text { categorias de } \\
\text { riscos, definir } \\
\text { parâmetros e } \\
\text { estabelecer } \\
\text { estratégia para a } \\
\text { gerência de risco }\end{array}$ & $\begin{array}{l}\text { Desenvolver o } \\
\text { Plano de } \\
\text { Gerenciament } \\
\text { o de Riscos }\end{array}$ & $\begin{array}{l}\text { Estabelecer o } \\
\text { escopo da gerência } \\
\text { de risco }\end{array}$ & \\
\hline $\begin{array}{l}\text { Identificação de } \\
\text { riscos }\end{array}$ & $\begin{array}{l}\text { Identificação } \\
\text { de riscos }\end{array}$ & $\begin{array}{l}\text { Identificar } \\
\text { riscos }\end{array}$ & $\begin{array}{l}\text { Identificar e } \\
\text { Avaliar os } \\
\text { Riscos }\end{array}$ & $\begin{array}{l}\text { Identificar } \\
\text { Riscos }\end{array}$ & $\begin{array}{l}\text { Identificar } \\
\text { riscos }\end{array}$ \\
\hline $\begin{array}{l}\text { Análise } \\
\text { qualitativa e } \\
\text { quantitativa de } \\
\text { riscos }\end{array}$ & $\begin{array}{l}\text { Análise e } \\
\text { Avaliação de } \\
\text { riscos }\end{array}$ & $\begin{array}{l}\text { Priorizar, estimar } \\
\text { e classificar } \\
\text { riscos }\end{array}$ & $\begin{array}{c}\text { Identificar e } \\
\text { Avaliar os } \\
\text { Riscos }\end{array}$ & $\begin{array}{l}\text { Analisar e } \\
\text { priorizar } \\
\text { riscos }\end{array}$ & $\begin{array}{l}\text { Analisar } \\
\text { riscos }\end{array}$ \\
\hline $\begin{array}{l}\text { Planejamento } \\
\text { de respostas a } \\
\text { riscos }\end{array}$ & $\begin{array}{l}\text { Tratamento de } \\
\text { riscos }\end{array}$ & $\begin{array}{l}\text { Desenvolver } \\
\text { Planos de } \\
\text { redução de risco }\end{array}$ & $\begin{array}{l}\text { Identificar e } \\
\text { Avaliar os } \\
\text { Riscos }\end{array}$ & $\begin{array}{l}\text { Definir a } \\
\text { Estratégia para a } \\
\text { gerência de } \\
\text { Risco }\end{array}$ & $\begin{array}{l}\text { Planejar } \\
\text { riscos }\end{array}$ \\
\hline $\begin{array}{l}\text { Monitoramento } \\
\text { e controle de } \\
\text { riscos }\end{array}$ & $\begin{array}{l}\text { Monitoramento } \\
\text { e análise crítica }\end{array}$ & $\begin{array}{l}\text { Implementar } \\
\text { Planos de } \\
\text { redução de risco }\end{array}$ & $\begin{array}{l}\text { Monitorar o } \\
\text { Status do } \\
\text { Projeto }\end{array}$ & $\begin{array}{l}\text { Definir } \\
\text { Métricas para } \\
\text { riscos, implementar } \\
\text { a estratégia da } \\
\text { gerência de risco e } \\
\text { avaliar os } \\
\text { resultados da } \\
\text { estratégia da } \\
\text { gerência de risco }\end{array}$ & $\begin{array}{l}\text { Acompanhar e } \\
\text { Controlar } \\
\text { riscos }\end{array}$ \\
\hline $\begin{array}{l}\text { Comunicação } \\
\text { implícita }\end{array}$ & $\begin{array}{l}\text { Comunicação e } \\
\text { consulta }\end{array}$ & $\begin{array}{l}\text { Comunicação } \\
\text { implícita }\end{array}$ & $\begin{array}{l}\text { Comunicação } \\
\text { implícita }\end{array}$ & $\begin{array}{l}\text { Comunicação } \\
\text { implícita }\end{array}$ & $\begin{array}{l}\text { Comunicação } \\
\text { implícita }\end{array}$ \\
\hline
\end{tabular}

Fonte: Adaptado de Rocha e Belchior (2004), Machado (2002), Gusmão e Moura (2005), PMI (2004) e 
Ferreira (2006).

Rocha e Belchior (2004) concluíram que o gerenciamento de riscos nos modelos estudados (PMBoK, CMMI, e RUP) está em consonância em seus aspectos essenciais, não havendo nenhuma incompatibilidade fundamental entre eles. Embora Machado (2002) tenha abordado os modelos PMBoK, CMMI e ISO/IEC 12207 - ISO/IEC 155045, considerou o PMBoK mais abrangente e em concordância com os objetivos pretendidos. Gusmão e Moura (2005) mostraram a similaridade entre os processos de Gerência de Riscos em projetos de software do CMMI, ISO/IEC 12207 - ISO/IEC 15504-5 e RUP. Segundo Gusmão e Moura (2005: 240), “com relação às atividades que compõem o processo de Gerência de Riscos, na literatura da área de Engenharia de Software, parece haver um consenso".

O gerenciamento de riscos do PMBoK, por se apresentar mais abrangente e em concordância com os objetivos propostos, é, portanto, adequado ao que se pretende, necessitando apenas explicitar a atividade de comunicação dos riscos, tomando-a emprestada da norma AN/NZS 4360:2004:

- Planejar a gerência de risco: decidir como abordar e executar as atividades de gerenciamento de riscos de um projeto e fornecer tempo e recursos e para estabelecer uma base acordada de avaliação deles.

- Identificar riscos: levantar todas as possibilidades existentes no projeto.

- Analisar riscos qualitativamente e quantitativamente: caracterizar os aspectos mais importantes, categorizando e priorizando os riscos, definindo as probabilidades de ocorrerem e os impactos, caso ocorram.

- Planejar respostas aos riscos: desenvolver opções e determinar ações para aumentar as oportunidades e reduzir as ameaças aos objetivos do projeto.

- Monitorar e controlar riscos: identificar, analisar e planejar os riscos recémsurgidos, os já identificados, re-analisar os existentes, monitorar as condições de acionamento de planos de contingência, monitorar os riscos residuais e revisar a execução de respostas a eles enquanto avalia sua eficácia.

- Comunicar os riscos: comunicar e consultar as partes envolvidas em cada etapa do processo de gerenciamento de riscos e em relação ao processo como um todo.

Como argumentado por Gusmão e Moura (2005: 241), em se tratando da engenharia de software, "de uma forma geral, os modelos e métodos relacionados ao processo de Gerência de Riscos utilizam estas atividades”.

\section{COMPARTILHAMENTO DO CONHECIMENTO NO GERENCIAMENTO DE RISCOS EM PROJETOS}

Segundo Neef (2005), a chave para um gerenciamento proativo de riscos repousa na habilidade da organização em mobilizar o conhecimento e perícia de seus empregados 
de forma que os líderes organizacionais possam garantir que eles consigam informações rápidas e acuradas sobre um fato potencialmente perigoso. De fato, alguns especialistas concordam que uma organização não pode gerenciar riscos hoje sem gerenciar seu conhecimento.

Murch (2001), citado por Cai et al. (2004), relata que corporações que utilizam a gestão do conhecimento para gerenciamento de projetos podem esperar aumento de $40 \%$ da produtividade no desenvolvimento de aplicações em tecnologia da informação. O gerenciamento do conhecimento é a única forma de prover as outras pessoas com a experiência que é conhecida pela organização (CAI et al., 2004).

Alguns autores, dentre eles Nonaka e Takeuchi (1997), vinculados ao tratamento do tema gestão do conhecimento, enfatizam a organização baseada na estruturação por projetos. Uma abordagem orientada a projetos pode ser enriquecida com elementos específicos de gestão do conhecimento, uma vez que permitiria que novas iniciativas organizacionais originais pudessem ser reiniciadas, com aproveitamento parcial ou total das experiências já vividas, tanto por parte das equipes dos projetos anteriores (conhecimento tácito), quanto por parte do conhecimento que ficou estruturado ao longo do desenvolvimento dos projetos concluídos ou ainda em andamento (conhecimento explícito) (GATTONI, 2004).

As formas como isso pode ocorrer devem envolver a utilização da gestão do conhecimento com ênfase no elemento humano, o grande receptáculo do conhecimento nas organizações, juntas de um suporte tecnológico e gerencial.

Segundo Muñoz-Seca e Rivelora (2004), as técnicas de gestão de projeto se desenvolveram para manejar a complexidade dos grandes projetos, entretanto essa perspectiva mudou no final do século XX. A maioria dos projetos que as empresas desenvolve de forma inovadora, para serem competitivas, caracteriza-se pela elevada incerteza.

O processo mais comum desses projetos "[...] é que se sabe o que se faz na medida em que se vai fazendo” (Muñoz-Seca; Rivelora, 2004: 63). Para os autores, a carência de conhecimentos adequados sobre um projeto leva-o a ter fases exploratórias, o que compromete o resultado final pelos problemas de tempo e qualidade. Uma forma de minimizar isso é "criar um contexto em que se identifiquem, absorvam e usem os conhecimentos o mais rápido possível para poder ser no mesmo momento em que se geram pela ação de qualquer dos agentes implicados no projeto" (Muñoz-Seca; Rivelora, 2004: 71).

Segundo Cooper (2003), sistemas de gestão do conhecimento atuam na redução de riscos pelo ganho e pelo processamento de informação relevante e pelo encapsulamento de conhecimento de grande variedade de fontes internas e externas.

Segundo Muñoz-Seca e Rivelora (2004), para projetos que geram conhecimento, sobretudo os de alta incerteza, há a necessidade de gerir não somente o projeto propriamente dito como também os processos laterais de geração de conhecimento. Essa gestão deve conseguir que, uma vez terminado o projeto, a organização tenha adquirido os ativos de conhecimento da melhor forma para ser competitiva. Os autores fazem referência à "centrifugação" do conhecimento às organizações ou projetos futuros que 
recebem os agentes participantes do projeto ao seu final.

Pretorius e Steyn (2005) concordam, afirmando que a performance de um projeto pode ser incrementada por meio do compartilhamento das melhores práticas e informações em tempo real. Assim como Muñoz-Seca e Rivelora (2004), para Pretorius e Steyn (2005) a lições aprendidas durante o ciclo de vida de um projeto podem prever a repetição de erros no mesmo projeto ou em outros similares e podem reduzir o custo e o tempo do retrabalho. Além disso, a documentação sistemática de erros e problemas potenciais ajuda a reduzir riscos e boas idéias geradas em um projeto podem ser transferidas para projetos futuros. Também segundo Leopoldino (2004), alguns estudos e padrões fazem referência ao uso do conhecimento retrospectivo acumulado em gerência de riscos na prática dos projetos. A parte relevante desse conhecimento sobre os riscos pode ser utilizada como subsídio para a tomada de decisões atuais e futuras.

Para Cai et al. (2004), o conhecimento existente na organização pode ajudar a mitigar riscos por permitir que problemas, na fase inicial do projeto, sejam expostos e entendidos. Há a necessidade de haver um repositório central de informações de projetos para que outros possam se beneficiar. Este corpo de conhecimentos também pode conter métricas de performance de projetos anteriores e técnicas usadas. Uma análise dos dados para definir padrões de sucesso ou fracasso é importante se destacar, entretanto não se deve centrar apenas em números e sempre aplicar o senso comum, assim as organizações podem se fortalecer por meio de mecanismos de feedback e experiências (CAI et al., 2004).

Neef (2005) descreve algumas técnicas e sistemas que estão sendo usadas nas organizações no que o autor chama de gerenciamento integrado de riscos e conhecimento:

- Mapeamento do conhecimento: é o uso de uma matriz de responsabilidade onde os empregados que são responsáveis por tomar decisões são mapeados e postos juntos eletronicamente para que responsáveis de projetos possam acessá-los.

- Comunidades de práticas: as comunidades são identificadas e os membros dessas redes são encorajados a reunir e transmitir, em uma base formal, idéias a respeito de potenciais atividades de risco, capturar lições aprendidas, trocarem idéias e compartilhar experiências sobre o tema.

- Especialistas “Hard-tagging”: é um processo de gerenciamento do conhecimento que combina mapeamento do conhecimento com um processo formal de aconselhamento quando empregados experientes são identificados ou marcados. Tornar a informação e experiência disponíveis para os líderes de uma empresa durante a eminência ou ocorrência de um risco é crítico para o processo de tomada de decisão no gerenciamento de risco.

- Aprendizado: é um contínuo e dinâmico processo de compartilhamento e aprendizado sempre presente na organização. Um dos grandes benefícios de um processo de análise de pós-acidente e continuo aprendizado é que os empregados digerem lições aprendidas de erros anteriores e a resolução do problema não requer "reinventar a roda”. 
- Cultura de compartilhar conhecimento: é o conceito que valores e expectativas de comportamentos éticos precisam ser amplamente comunicados e efetivamente difundidos na organização. Significando que há necessidade de ter um processo que encoraje compartilhar idéias e a identificação imediata de riscos.

- Monitoramento e divulgação do desempenho: a razão para o sucesso do conhecimento esta na necessidade de medir, monitorar e divulgar o desempenho organizacional, quando informações não financeiras são usadas para predizer o sucesso futuro de uma empresa.

- Envolvimento dos interessados e comunidade: a comunicação e compartilhamento das políticas da empresa não só para os empregados, mas também para os demais interessados na empresa e para a comunidade. Também ajuda os líderes empresariais a responder imediatamente a estas partes externas prevendo 0 desenvolvimento de conflitos ou incidentes.

- Pesquisa e análise de negócios: é a habilidade da organização em reunir informação, desenvolver uma capacidade de pesquisa e analise do conhecimento no intuito de encontrar, organizar e distribuir informação de fontes internas e externas sobre atividades políticas, culturais e legais.

Por meio de um levantamento na literatura não se encontrou uma confluência mais aprofundada entre os dois temas aqui tratados, compartilhamento do conhecimento e gerenciamento de riscos em projetos. A ausência de uma ligação profunda entre esses dois temas levou a um estudo exploratório sobre a questão da pesquisa, buscando informações, sobretudo em estudos anteriores, como foram apresentados.

\section{METODOLOGIA}

O tipo de estratégia adotado nesta pesquisa foi o estudo de casos, com propósito exploratório. Conforme Yin (2001), a preferência pelo uso de estudo de casos deve ser escolhida quando o estudo suporta eventos em que é possível se fazer observações diretas, aplicação de questionários e entrevistas. O autor ressalta que o estudo de casos é comumente um método de pesquisa específico para estudos exploratórios, onde se tem pouco conhecimento sobre o assunto, mas que há possibilidade de seu uso em estudos descritivos e causais. A forma de coleta de dados deve adaptar-se aos objetivos e às especificidades de cada estudo.

Apesar da análise de um único ou poucos casos fornecer uma base frágil para a generalização, os propósitos do estudo de caso não são os de proporcionar o conhecimento preciso das características de uma população, mas sim proporcionar uma visão global do problema ou identificar possíveis fatores que o influenciam ou são por ele influenciados (Gil, 2008).

O universo de pesquisa é formado por empresas da indústria de software do Brasil, sendo a unidade de análise empresas de software capacitadas para processos reconhecidos pelo mercado sobre a maturidade no gerenciamento de projetos de desenvolvimento de software e que refletem na qualidade do produto resultante, como o 
CMMI-SW e MPS.BR (Melhoria do Processo do Software Brasileiro). A unidade de observação foram os membros de equipes de desenvolvimento de projetos de software dessas organizações, que atuam na cidade de Belo Horizonte, Minas Gerais. Fazem parte deste grupo todos os profissionais que trabalham ligados à gestão do desenvolvimento de projetos, tais como gerentes de projeto, desenvolvedores de software, analistas de sistemas e técnicos em informática. A pesquisa de campo foi realizada em duas empresas de Belo Horizonte, Minas Gerais, que têm como atividade o desenvolvimento de software.

Optou-se por coletar as informações quantitativas e qualitativas, por meio de três instrumentos de coleta, de forma a proceder à triangulação de fontes de informação: um questionário e um roteiro de entrevista semi-estruturada e pesquisa bibliográfica.

O questionário foi elaborado tomando-se por base os instrumentos de compartilhamento do conhecimento que foram relacionados às atividades de gestão de riscos em projetos de software, conforme levantados na revisão da literatura. Os aspectos comportamentais facilitadores do compartilhamento do conhecimento foram relacionados ao processo de gestão de riscos, conforme levantados na literatura, sob o ponto de vista individual e da equipe de projeto. O questionário contém também questões como idade, cargo, gênero, tempo de experiência do profissional e nível de escolaridade que permitiram caracterizar a amostra.

Foram coletadas e analisadas as percepções e opiniões dos participantes da pesquisa sobre suas práticas nas empresas em que atuam referentes ao gerenciamento de riscos e o compartilhamento de riscos em projetos, bem como a relação entre os dois temas.

Para tanto, os questionários foram elaborados utilizando-se itens com resposta em escala de classificação Likert de 5 pontos, que permite que um valor numérico seja dado a uma opinião (Collis; Hussey, 2005), o que permitiu transformar os resultados em valores numéricos. Adicionalmente, foi também adotada uma abordagem qualitativa complementar, por meio de entrevista em profundidade, buscando esclarecer aspectos mais subjetivos dos fenômenos observados, eventualmente não capturados no questionário aplicado e/ou não observados através da revisão de literatura.

Este trabalho adotou como critério de seleção a amostra não-probabilística selecionada por tipicidade, o que diminui a possibilidade de inferir para o todo os resultados obtidos (Marconi e Lakatos, 2007). A escolha da amostra intencional levou em conta os indivíduos que, segundo a experiência e o entendimento do pesquisador, são sujeitos sociais que detêm um conjunto de conhecimentos, experiências e relacionamentos que se pretendia interpretar na pesquisa e, neste sentido, pudessem contribuir para o objetivo do estudo.

A aplicação da pesquisa ocorreu por meio da aplicação de questionários a 90 membros de equipes de projeto de software, com o retorno de 69 questionários válidos. Após a aplicação dos questionários, 5 pessoas que responderam aos questionários, 2 de uma empresa e 3 de outra, foram selecionadas para uma entrevista, tomando como base o envolvimento dessas pessoas no gerenciamento de riscos em projetos. 


\section{ANÁLISE E INTERPRETAÇÃO DOS RESULTADOS}

Por meio do questionário, foi apurado grande domínio de trabalhadores masculinos na indústria de software, na amostra pesquisada (81,2\%). Pouco mais da metade (50,7\%) dos trabalhadores possui idade de 25 a 35 anos. Quanto à formação, parte significante dos trabalhadores da indústria de software possui uma especialização $(39,1 \%)$ ou o $3^{\circ}$ grau $(42,0 \%)$. A maior proporção dos trabalhadores está na empresa entre 1 e 3 anos (63,8\%) e, referente ao cargo ocupado, a maioria dos trabalhadores é analista (65,2\%).

Nas próximas seções são detalhados os resultados obtidos no estudo, alinhados com os dois objetivos principais propostos no artigo.

\subsection{Atividades de gerenciamento de riscos em projetos de software.}

Ao tentar identificar as atividades de gerenciamento de riscos em projetos nas empresas estudadas, verificou-se que a maioria dos trabalhadores $(65,2 \%)$ participa de alguma atividade de gerenciamento de risco, com tendência dos ocupantes de cargo de gerência, coordenação ou liderança em participar dessas atividades. Os entrevistados, quando perguntados sobre como é o método de gerenciamento de riscos em projetos nas empresas, responderam apontando, no geral, para as atividades de identificação, análise, resposta e monitoramento e controle, como foi descrito por Rocha e Belchior (2004), Machado (2002), Gusmão e Moura (2005), PMI (2004) e Ferreira (2006). Na entrevista, não ficaram evidenciadas as atividades de comunicação e planejamento do gerenciamento. Entretanto, no tocante à comunicação dos riscos, como citado por esses autores, esta é realizada de forma implícita durante as várias atividades do gerenciamento. No caso da atividade de planejamento do gerenciamento, verificou-se que esta se confunde com o processo geral de planejamento do projeto.

\subsection{Instrumentos de compartilhamento nas atividades de gerenciamento de riscos em projetos de software.}

Os respondentes do questionário afirmam que as empresas dispõem de instrumentos ou métodos formais para compartilhar conhecimento (63,8\%).

No intuito de restringir e facilitar a análise e conclusões posteriores considerou-se os instrumentos classificados em primeiro e segundo lugares para cada atividade do processo de gerenciamento de riscos em projetos.

O QUADRO 4 apresenta os principais instrumentos que contribuem para cada atividade do gerenciamento de riscos por meio da ordenação que foi realizada a partir das porcentagens de concordância. 


\section{QUADRO 4}

Ordenação dos principais instrumentos que contribuem para cada atividade do gerenciamento de riscos em projeto

\begin{tabular}{|c|c|c|c|c|c|c|}
\hline \multirow[t]{2}{*}{ Instrumentos } & \multicolumn{6}{|c|}{ Atividades do Gerenciamento de Risco em projeto } \\
\hline & $\begin{array}{l}\text { Planejamento } \\
\text { do } \\
\text { gerenciamento }\end{array}$ & Identificação & Análise & $\begin{array}{l}\text { Planejamento de } \\
\text { respostas }\end{array}$ & $\begin{array}{l}\text { Monitoramento } \\
\text { e controle }\end{array}$ & Comunicação \\
\hline Narrativas & $\mathbf{1}^{\mathbf{o}}$ & $\mathbf{1}^{\mathbf{o}}$ & & & $2^{\mathbf{o}}$ & $\mathbf{1}^{\mathbf{0}}$ \\
\hline Repositórios & $2^{0}$ & & $\mathbf{1}^{\mathbf{o}}$ & $2^{0}$ & & $2^{\mathbf{o}}$ \\
\hline Cenários & & $2^{\mathbf{o}}$ & & $\mathbf{1}^{\mathbf{o}}$ & & \\
\hline Equipes & & & $2^{o}$ & & $\mathbf{1}^{\mathbf{o}}$ & \\
\hline
\end{tabular}

Fonte: Dados da pesquisa.

Com esses resultados, é possível verificar quais instrumentos mais contribuem para cada atividade de gerenciamento de riscos em projetos. Observa-se, assim, que os instrumentos "Narrativas" e "Repositórios" apresentam-se ocupando o primeiro ou segundo lugares nessas atividades. Os instrumentos referentes às comunidades de práticas e bancos de competências não foram classificados nos primeiros lugares, portanto, os participantes da pesquisa não concordam que possam contribuir em maior escala para as atividades de gerenciamento de riscos em projetos.

\subsection{Aspectos facilitadores no gerenciamento de riscos em projetos de software}

A maioria dos trabalhadores (73,9\%) afirma que são formalmente incentivados pelas empresas a compartilhar o que eles sabem. Entretanto, conforme apurado na entrevista, foi demonstrada a inexistência de um programa formal e estruturado nas empresas para a gestão do conhecimento ou efetiva aplicação deste a todos os trabalhadores.

Para a verificação de quais aspectos facilitadores são considerados os mais importantes na contribuição ao compartilhamento de riscos, para os trabalhadores das empresas estudadas, foi elaborado o GRAF. 1 que apresenta os escores médios que os respondentes atribuíram para cada assertiva. Os maiores escores médios indicam as maiores concordâncias, consequentemente maior importância da assertiva. No intuito de restringir e facilitar a análise considerou-se aqueles fatores que apresentam os três maiores escores médios. 


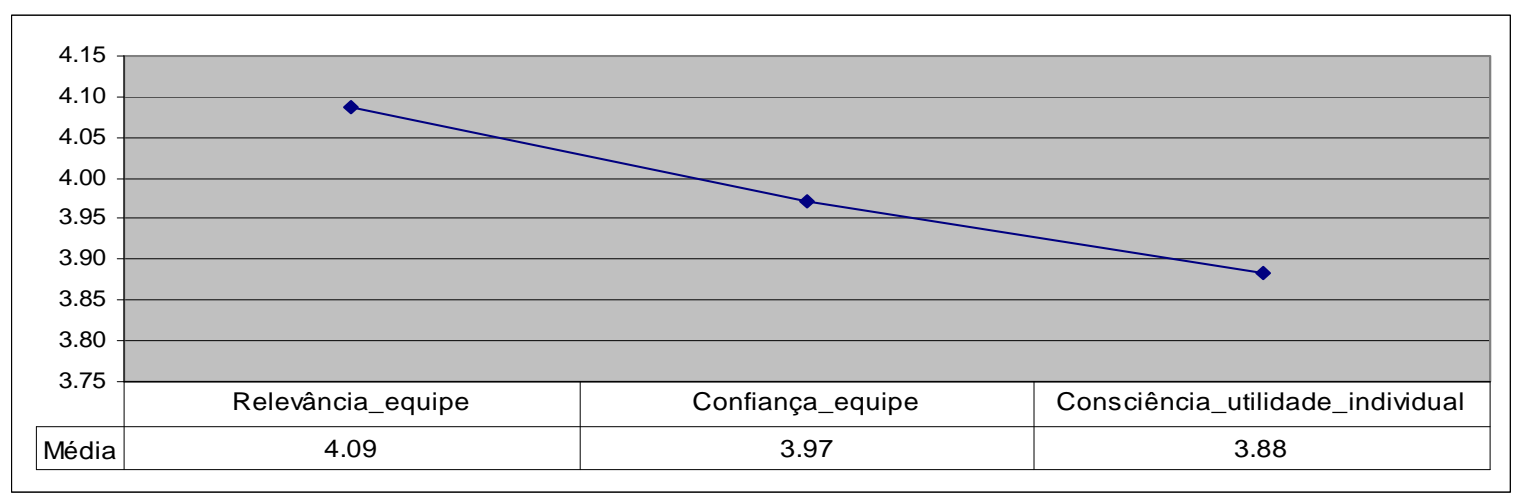

GRÁFICO 1 - Importância dos aspectos facilitadores para o gerenciamento de riscos Fonte: Dados da pesquisa.

É possível observar, por meio do GRAF. 1, que os facilitadores "Relevância_equipe”, "Confiança_equipe”, "Consciência_utilidade_individual” (maiores escores médios) são percebidos como os que mais contribuem para o gerenciamento de riscos.

Além disto, na entrevista foram obtidas importantes declarações dos entrevistados que sustentam a idéia de forte evidência empírica das ligações entre os aspectos comportamentais facilitadores do compartilhamento do conhecimento e o gerenciamento de riscos em projetos de software.

\section{CONCLUSÃO}

O estudo de caso apontou as atividades de gerenciamento de riscos em projetos de software que, na opinião e percepção dos participantes pesquisados, são executadas nas empresas em que trabalham, estando os resultados em consonância com a teoria revista. Aproximadamente 64\% dos respondentes afirmam que as empresas dispõem de instrumentos ou métodos formais para compartilhar conhecimento.

Os resultados do estudo apontam para certos aspectos comportamentais que podem influenciar favoravelmente para o compartilhamento de conhecimento sobre riscos em projetos de software. Em organizações que lidam com projetos podem ser desenvolvidas atividades que levem o indivíduo a perceber que há ganhos relevantes para ele ou sua equipe de projeto ao compartilharem conhecimento sobre riscos; que este conhecimento quando compartilhado não trará danos a ele ou para sua equipe e que este conhecimento pode ter utilidade para outras pessoas na organização. Desta forma, espera-se transpor barreiras e obstáculos como a pouca disposição em compartilhar e a falta de ambientes ou meios de interação, tanto no nível individual quanto organizacional.

Por meio do estudo realizado é possível entender como certos instrumentos podem contribuir para o compartilhamento do conhecimento sobre riscos em cada atividade de 
gerenciamento de riscos em projetos de software. Segundo os participantes do estudo, a transmissão das experiências das pessoas e a recuperação e uso dos registros e documentos sobre gerenciamento de riscos podem garantir que o nível, tipo e a visibilidade do gerenciamento de riscos estejam de acordo com o risco e a importância do projeto em relação à organização e forneçam tempo e recursos suficientes para as atividades de gerenciamento de riscos. Também as experiências das pessoas sobre riscos e a construção de cenários, protótipos e simulações sobre situações de risco são as categorias mais importantes que podem garantir o sucesso na execução do levantamento de todas as possibilidades de riscos existentes no projeto. Para melhor caracterizar os aspectos mais importantes de cada risco, categorizando e priorizando-os, definindo adequadamente as probabilidades de ocorrerem e os impactos, caso ocorram, a recuperação e uso dos registros e documentos sobre gerenciamento de riscos e as diferentes fontes de conhecimento e visões sobre o assunto podem ser importantes para potencializar a execução da atividade de análise. A construção de cenários, protótipos e simulações sobre situações de risco e a recuperação e uso dos registros e documentos sobre gerenciamento de riscos também podem contribuir para desenvolver as melhores opções e determinar ações adequadas para aumentar as oportunidades e reduzir as ameaças aos objetivos de um projeto. A vivência de profissionais experientes bem como de pessoas com diferentes fontes de conhecimento e visões sobre como monitorar e controlar riscos colabora para identificar, analisar e planejar riscos recém-surgidos, acompanhar os já identificados, re-analisar os existentes; monitorar as condições de acionamento de planos de contingência, os riscos residuais e revisar a execução de respostas a eles enquanto se avalia sua eficácia. E finalmente, para melhor comunicar e consultar as partes envolvidas internas e externas em cada etapa do processo de gerenciamento de riscos e em relação ao processo como um todo, podem ser aplicadas as experiências de profissionais, a recuperação e o uso dos registros e documentos sobre gerenciamento de riscos.

Na percepção dos participantes da pesquisa é possível inferir, sem generalizar, que, ao combinar certos aspectos comportamentais ao uso de instrumentos que ajudam no compartilhamento do conhecimento, as organizações podem usufruir de melhorias no processo de gerenciamento de riscos, sinalizando para uma relação positiva entre o compartilhamento do conhecimento e o gerenciamento de riscos em projetos.

Deve-se considerar como limitação que a amostra pesquisada possui tamanho restrito e foi construída de forma não-probabilística, não permitindo generalizações. Outro ponto se refere a um viés típico da forma como o levantamento foi conduzido: ao se disporem, de forma livre, a participar da pesquisa, os respondentes e entrevistados já demonstram, por este ato, uma atitude solícita e colaborativa, uma predisposição ao compartilhamento de conhecimento. O terceiro ponto limitante que merece consideração é o fato de esta ser uma pesquisa exploratória. Os métodos de coleta e de análise de dados refletem, portanto, esta situação. Outro ponto a considerar é que na pesquisa foram coletadas as opiniões e percepções dos membros das equipes de projetos sobre a realização de atividades de gerenciamento de riscos em projetos, do compartilhamento de conhecimento sobre riscos e da relação dos dois temas, sem confirmações concretas e materiais da existência destas. Além disso, poucos foram os trabalhos encontrados na literatura sobre uma ligação entre os temas compartilhamento de conhecimento e gerenciamento de riscos em projetos. 
Ao mostrar as limitações naturais de um estudo de caráter exploratório, se dá condição de ampliar os trabalhos em torno do tema e estudos mais conclusivos, utilizando métodos confirmatórios, poderão ser realizados com o intuito de ampliar o entendimento das questões levantadas nesta pesquisa. Buscar conhecer a realidade de empresas com diferentes condições de atividades, inclusive geográficas, também completaria a pesquisa aqui descrita. Assim, desenvolver um estudo comparando as práticas e percepções de profissionais de empresas que lidam com projetos em ramos diferentes de atividades, é outro passo para o entendimento mais abrangente da questão. Recomenda-se ainda que seja conduzida uma pesquisa que permita a coleta de evidências diretas e concretas (listas de riscos, planos de mitigação e contingências, atas de reunião, ocorrência de palestras, fóruns de conhecimento, etc) sobre a execução das atividades de gerenciamento de riscos em projetos, o compartilhamento de riscos em projetos, bem como a relação entre os temas.

\section{REFERÊNCIAS}

Alencar, A. J., \& Schimitz, E. A. (2005). Análise de risco em gerência de projetos. Rio de Janeiro: Brasport.

Argote, L., \& Ingram, P. (2000). Knowledge Transfer: A Basis for Competitive Advantage in Firms. In: Organizational Behavior and Human Decision Processes, 82 (1): 150-169. Recuperado em: 19 de agosto, 2006, de http://www.idealibrary.com/

Bukowitz, W. R, \& Williams, R. L. (2002). Manual de Gestão do Conhecimento. São Paulo: Bookman.

Cai, Yongxue et al. Identifying Best Practices in Information Technology Project Management. 2004. Recuperado em 27 de julho, 2006, de http://www.pmforum.org/library/papers/2004/itpractices.pdf

Collis, J., \& Hussey, R. (2005). Pesquisa em administração: um guia prático para alunos de graduação e pós-graduação (2a ed.). Porto Alegre: Bookman.

Cooper, L. P. (2003). A research agenda to reduce risk in new product development through knowledge management: a practitioner perspective. Journal of Engineering and Technology Management, 20 (1-2): 117-140.

Davenport, T. H., \& Prusak, L. (1998). Conhecimento empresarial: como as organizações gerenciam o seu capital intelectual. Rio de Janeiro: Campus.

Ferreira, G. AS/NZS 4360:2004 Australian Standard for Risk Management. Jan., 2006. Recuperado em 17 de junho, 2006, de http://www.checkuptool.com.br/artigo_14.htm

Fumsoft. Sociedade Mineira de Software. Empresas de TI de Belo Horizonte alcançam a excelência na produção de software.Recuperado em 7 julho, 2007 , de http://www.fumsoft.softex.br/fum_noticias.shtm

Gattoni, R. L. C. (2004). Gestão do conhecimento aplicada à prática da gerência de 
projetos. Belo Horizonte: C/ Arte.

GIL, A. C. (2008). Como elaborar projetos de pesquisa (4a ed.). São Paulo: Atlas.

Gusmão, C. M. G. de, \& Moura, H. P. de. (2005). Gestão de Riscos para Ambientes de Múltiplos Projetos de Software: Teoria e Prática. In C. M. G. de Gusmão, \& H. P. de Moura. IV Escola Regional de Informática de Minas Gerais (Cap. 7, pp. 229-277). Belo Horizonte: Sociedade Brasileira de Computação.

Kendrick, T. (2003). Identifying and Managing Project Risk: Essential Tools for Failure-Proofing Your Project (1a ed.). New York, NY, USA: Amacom.

Kerzner, H. (2003). Project Management: A Systems Approach to Planning, Scheduling, and Controlling (8a ed.). New Jersey, USA: John Wiley \& Sons, 2003.

Kerzner, H. (2006). Gestão de projetos: as melhores práticas. Porto Alegre: Bookman.

Leonardi, S. M. (2005). O compartilhamento de conhecimento em equipes de venda: um estudo exploratório no setor químico. Dissertação de Mestrado em Administração de Empresas, Centro Universitário Álvares Penteado - UNIFECAP, São Paulo, Brasil.

Leopoldino, C. B. (2004). Avaliação de Riscos em Desenvolvimento de Software. Dissertação de Mestrado em Administração, Universidade Federal do Rio Grande do Sul, Porto Alegre, Brasil.

Machado, C. Â. F. (2002). A-Risk: Um método para identificar e quantificar risco de prazo em projetos de desenvolvimento de software. Dissertação de Mestrado em Ciências, Programa de Pós-Graduação em Informática Aplicada, Pontifícia Universidade Católica do Paraná, Curitiba, Brasil.

Magnani, M. (2004). Identificação de fatores críticos de sucesso para a formulação de estratégias que minimizem a perda de competência organizacional de um centro de $P \& D$ agropecuário. Tese de Doutorado em Engenharia de Produção, Programa de PósGraduação em Engenharia de Produção do Departamento de Engenharia de Produção e Sistemas, Universidade Federal de Santa Catarina, Florianópolis, Brasil.

Marconi, M. de A., \& Lakatos, E. M. (2007). Metodologia do trabalho científico: procedimentos básicos, pesquisa bibliográfica, projetos e relatório, publicações e trabalhos científicos (7a ed.). São Paulo: Atlas.

Muñoz-seca, B., \& Rivelora, J. (2005). Transformando Conhecimento em Resultados. São Paulo: Clio.

Neef, D. (2005). Managing corporate risk through better knowledge management. Chester, New Jersey, USA. The Learning Organization. 12 (2): 112-124. Recuperado em 10 setembro, 2009, de www.emeraldinsight.com/0969-6474.htm

Nonaka, I., \& Takeuchi, H. (1997). Criação de conhecimento na empresa: como as empresas japonesas geram a dinâmica da inovação. Rio de Janeiro: Campus.

Pretorius, C. J., \& Steyn, H. (2005). Knowledge management in project environments. In: South Africa Journal of Business Management. Recuperado em 3 abril, 2006, de http://pkp.sfu.ca/ojs/demo/testdrive/index.php/sadness/article/view/202

Probst, G., Raub, S., \& Romhardt, K. (2002). Gestão do Conhecimento: os elementos 
construtivos do sucesso. Porto Alegre: Bookman.

PROJECT MANAGEMENT INSTITUTE. (2004). A Guide to The Project Management Body of Knowledge. Four Campus Boulevard, Newtown Square, PA, EUA,. 405p.

Rocha, P. C., \& Belchior, A. D. (2004). Mapeamento do Gerenciamento de Riscos no PMBOK, CMMI-SW e RUP. In: Simpósio Internacional de Melhoria de Processos de Software, 4, São Paulo. Recuperado em 22 maio, 2006, de http://www.simpros.com.br.

Schwalbe, K. (2002). Information Technology Project Management. Cambridge, MA: Course Technology.

SOFTWARE ENGINEERING INSTITUTE. CMMI for Systems Engineering/Software Engineering (CMMI-SE/SW), Staged Representation, Version 1.1, Technical report CMU/SEI-2002-TR-02. Pittsburgh, PA: Software Engineering Institute, Carnegie Mellon University, 2002.

Swebok, Guide for the Software Engineering Body of Knowledge, 2004 version, IEEE Computer Society, California, EUA.

Sveiby, K. E. (2001). A knowledge-based theory of the firm to guide in strategy formulation. Journal of Intellectual Capital, 2 (4), Recuperado em 23 maio, 2006, de http://www.sveiby.com/Portals/0/articles/Knowledgetheoryoffirm.htm

Tonet, H. C., \& Paz, M. G. T. (2006). Um Modelo para o Compartilhamento de Conhecimento no Trabalho. Revista de Administração Contemporânea, 10 (2): 75-94.

Vargas, R. V. (2000). Gerenciamento de projetos: estabelecendo diferenciais competitivos. Rio de Janeiro: Brasport.

Von krogh, G., \& Ichijo, K. N. I. (2001). Facilitando a criação de conhecimento: reinventando a empresa com o poder da inovação contínua. Rio de Janeiro: Campus.

YIN, R. K. (2005). Estudo de caso, Planejamento e métodos (3a ed.). Porto Alegre: Bookman. 
A contribuição do compartilhamento do conhecimento para o gerenciamento de riscos em projetos: um estudo na indústria de software 